\section{Leonardo DaVinci: The First Photographer?}

Bob Compton, Corresponding Editor

Some 350 years before the invention of photography, Leonardo DaVinci may very well have produced the first photograph - the Shroud of Turin. This extraordinary hypothesis attracted shroud experts Lynn Picknett and Clive Prince, and their efforts to prove or disprove this theory resulted in their recent book Turin Shroud. During their shroud investigations, the authors delved deeply into historical lore, piecing together diverse facts and opinions including those of such figures as the Knights of Templar, alchemists, secret societies, and even Pope Innocent VIII. The authors provided convincing evidence that the shroud could have been produced using photographic-like techniques that were within the identified capabilities of DaVinci. Most importantly, the authors were able to recreate shroud "look-alike" images with materials and techniques believed to be available to, or devised by, Leonardo DaVinci in the creation of the Turin Shroud.

\section{Why not a painting?}

Over the centuries there has been a continuing controversy over the shroud's authenticity - whether or not it was indeed the shroud used to cover the entombed Jesus Christ. The authors delved deeply into this controversy and described many of the scientific and not-so-scientific efforts used to ascertain how the image could have been formed. The opinions ranged widely, including many who believed that the image was the result of an ingenious painter, to those who considered the image to have a divine origin. The controversy took an interesting twist in 1988, when carbon dating tests identified that the shroud cloth was produced in the late 1300's. These test results stimulated renewed interest in determining alternate methods of image formation.

As noted by the authors, tests made by the Mccrone Research Institute using 1978 samples, identified the presence of iron oxide in the shroud image areas (see Microscopy Today Jan/Feb 1995). Because iron oxide was a common material used to formulate Venetian red, a paint common in DaVinci's time, the Mccrone test data supported the possible conclusion that paints were used in the fabrication of the image. However, it is the authors' claim that there are plausible explanations for the presence of iron oxide in the shroud cloth other than from the use of paint to form the image. Also they claim that other available evidence indicates that the shroud could not have been made using painting techniques.

If the shroud was not of divine origin and was not painted, then how could the image have been made? Some of the many possibilities proposed over the years ranged from a nuclear flash to chemical reactions resulting from rising vapors. After analyzing many of the plausible suggestions and hypotheses, the authors decided that one of the best possibilities was that the shroud was produced using photographic-like techniques. Other evidence led the authors to believe the hypothesis that the image was produced by DaVinci, probably in 1492 . To prove or disprove this these theory, the authors initiated a series of experiments in an attempt to create a shroud-like image.

\section{EDS to fit your Budget!}

Choose basic acquisition, fully quantitative or standardless analysis on either a Macintosh or IBM platform. Use your existing detector and pulse processor or buy a new complete system from us.

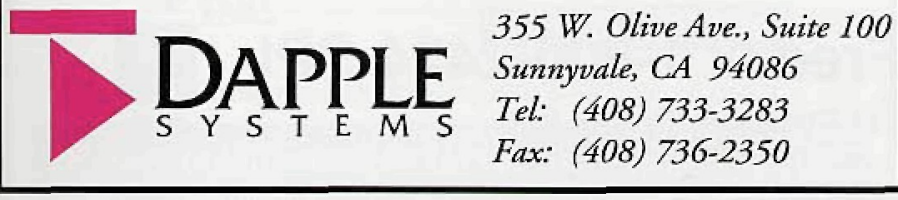

The Challenge

In creating what may have been the first photograph, DaVinci would have had to devise an appropriate imaging method, select and apply appropriate lightsensitive materials to an appropriate cloth, and devise methods of processing the exposed materials to create a final image that would have the mystical reality necessary to fool expert viewers.

\section{The Imaging Method}

The author's investigation led them to believe that a variation of the camera obscura (pinhole camera) was probably the imaging technique used by DaVinci. This, they feel, was reasonable in that there is historical evidence that knowledge of the camera obscura method of producing an image had been in existence for many years before DaVinci"s time. Thus the authors challenged themselves to devise an appropriate camera obscura technique that could produce a shroud-like image.

One of the first problems that authors had to resolve was the excessive time required to produce a life-sized image. When using a classical pinhole camera configuration, several days were required to form a sufficiently dark image. One possible option considered by the authors, was to intensely illuminate the subject using parabolic mirrors to concentrate the available sunlight. It is believed that Davinci was knowledgeable in the use of such mirrors and it was one of his options. Other experiments led the authors to believe that DaVinci added a small lens to the pinhole of a camera obscura. When the authors formed an image with the lens in place, the exposure time necessary to produce an effective image was reduced to reasonable value.

\section{Capturing the Image}

After considering many alternate methods of capturing an image on a cloth, the authors decided to run a series of experiments based on the idea that the shroud image might have been formed using an "invisible ink" process. Here the cloth would be coated with a suitable mixture, exposed, then heated to make the image visible.

The first problem was to select a suitable light sensitive material. Various materials were known to be light sensitive during DaVinci's day, as alchemists had noted that many plants, roots, and petals changed color when exposed to light. Also several inorganic materials including iron, copper and mercury salts were known to be light sensitive. Although not "officially" known in DaVinci's time, chromates were considered to be within his area of expertise and readily available to the authors. Thus, after many experiments, the authors achieved success by treating the cloth with a mixture of egg white, bichromate and urine. After exposure using the camera obscura technique, the treated, exposed cloth was placed in front of a fire, heated evenly, and washed. Not only did the resulting image have a scorched "shroud-like" appearance but all traces of the saltegg white mixture disappeared.

When analyzing the images they produced, the authors noted with dismay that several image anomalies occurred. One anomaly was a circular area in the center of the resulting image that looked like a miniature image of the lens itself. This dismay turned to one of relief when, upon further examination, a similar anomaly was observed in the shroud image. Other anomalies in the shroud image, such as image foreshortening and the absence of ear images also seemed to verify this method of shroud image generation. Thus the image produced by the authors had many characteristics of the shroud image, including the lack of pigmentation.

Although the authors noted that not all questions relating to the shroud image have been answered by their experimental results, they claim with confidence that the images they were able to produce bear many similarities to the shroud image. Because their images exhibit many of the shroud's puzzling anomalies, they are confident that similar techniques were used in the creation of the shroud image and that the shroud image was probably made by Leonardo DaVinci - the first photographer

The book Turin Shroud, written by Lynn Picknett \& Clive Prince, published by Harper Collins, is available in many bookstores. It not only identifies the practical techniques the authors used to produce shroud-like images but provides compelling background relating to the history and mystery of the shroud's ancestry. It is a book well worth reading. 


\section{McCRONE RESEARCH INSTITUTE}

2820 S. MiChIGAN A venue, ChICAGo, IL 60616-3292

McCrone Research Institute offers a variety of 5-day microscopy courses. Most of these are scheduled several times a year. Each offers a strong foundation in both theory and application and there is one light microscope for each student.

\section{New and Special Course Offerings}

Sample Preparation and Manipulation of Particles (501E) - Collection, isolation, manipulation, cleaning and mounting of single-particles $(<10$ $\mu \mathrm{m})$. Taught by Anna Teetsov of the McCrone Associates Laboratory and Skip Palenik.

Microscopy of Environmental Dust (530*) - Application of light microscopy to the analysis and identification of dust particles that are formed in domestic and industrial environments. Includes pollen, mold and pollutants.

Special Asbestos Problems (512*) - Advanced practical methods including identification of submicrometer fibers, quantitation, floor tile examination, dispersion staining and microchemcial testing.

Drug Identification (526*) - Optical crystallographic methods, microchemical testing and dispersion staining. Emphasis on street drugs.

Microscopical Study of Paints and Extenders (520B*) - Includes sectioning methods, epi fluorescence microscopy; identification of inorganic pigments and extenders, microchemical testing, pigment sublimation, optical crystallography and oil immersion techniques.

Heavy Minerals and Pollen in Soil Investigation $\left(510 \mathrm{~B}^{*}\right)$ - Isolation and microscopical characterization of heavy minerals and pollen from soil samples. Taught by Skip Palenik.

Computers in Microscopy $\left(701^{*}\right)$ - A survey of computer-assisted microscopy including basic principles, spreadsheets, databases, image editing and image processing. Requires basic knowledge of microscopy but no previous computer experience required.

\section{Electron Microscopy}

Scanning Electron Microscopy (402)

TEM Introduction (407A)

TEM Asbestos Analysis (407B*)

TEM SAED and EDS Techniques (408*)

\section{General Courses/Methodology}

Photomicrography (101)

Applied Polarized Light Microscopy (201)

Microchemical Analysis (207A)

Advanced Microchemical Analysis (207B*)

Identification of Small Particles (501A)

Advanced Small Particle Identification $\left(501 \mathrm{~B}^{*}\right)$

Computers in Microscopy (701*)

\section{Specialized Courses - Materials}

Pharmaceutical Microscopy (503)

Forensic Microscopy (504)

Polymer, Fiber \& Film Microscopy (505)

Microscopy \& Microchemistry of Polymers (505B*)

Microscopy for Art Conservators (506)

Fiber Identification (507*)

Heavy Minerals and Pollen in Soil Investigation (510B*)

Wood and Pollen Microscopy (511*)

Microscopy of Explosives (522*)

Mineral Identification (523)

Drug Identification (526*)

Forensic Examination of Building Materials (527B*)

Microscopical Study of Paints and Extenders (520B*)

\section{Asbestos/Environmental Courses}

Microscopical Identification of Asbestos (508A)

Advanced Asbestos Identification (508B ${ }^{*}$ )

Special Asbestos Problems (512*)

Asbestos Fiber Counting [NTOSH 582] (516)

Quantitative Asbestos Analysis (528*)

Microscopy of Environmental Dust (530*)

*Prerequisite: course 201 or equivalent; $207 \mathrm{~A}$ for $207 \mathrm{~B} ; 508 \mathrm{~A}$ for $508 \mathrm{~B}$ or $528 ; 407 \mathrm{~A}$ for $407 \mathrm{~B}$.

Tuition of $\$ 1050$ except courses 528 ( 3 days at $\$ 650), 516(\$ 850)$, 407A ( $\$ 1250)$, and 407B or 408 ( $\$ 1750)$.

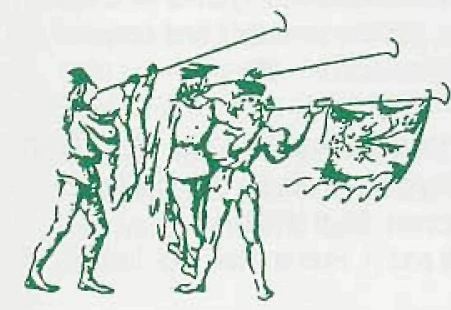

INTER/MICRO-95 celebrates the 47th Anniversary of these microscopy conferences at the Knickerbocker Hotel in Chicago, 10-13 July 1995. You will have the opportunity to come up-to-date on new instruments for, new techniques in, and new applications of, microscopy.

Microscopy can best be defined as any tool or technique enabling the microscopist to characterize, identify and study microscopic samples. This, therefore, includes all light and electron microscopes, microspectrophotometers, microprobes, automatic image analyzers and other microscopes based on x-rays, sound, protons, etc. Papers will cover new techniques for improving contrast, increasing resolution, as well as obtaining and recording more characterization data; also, how new techniques or new instruments are used in solving important problems. Most papers presented at INTER/MICRO-95 will be published in The Microscope, the official journal for these conferences. These are published in the order they are received throughout the year. A one-year subscription is $\$ 65$ (individuals paying personally, only $\$ 44$ ).

Contact Nancy Daerr at McCrone Research Institute, 312/842-7100 for information. 